تثخيص اضطراب الأتوية في الاليل الرابع DSM-4 والدليل الخامس DSM-5 للَاضطرابات العقلية (دراسة وصفية)

إعداد

الباحث / محمد رضا السيد محمد'

الإثراف

دانهى ضياء الدين عبد الحميد

مدرس بقسم العلوم النفسية

كلية التربية للطفولة المبكرة

جامعة القاهرة
أ. أدالد عبد الرازق النجار

أستاذ علم النفس عبد الرأف

ورئيس قسم العلوم النفسية

كلية التربية للطقولة المبكرة

جامعة القاهزة

\title{
مقدمة
}

اضطراب الذاتوية اضطراباً نمائياً عصبياً معقداً يرجع الفضل لمعرفته إلى الطبيب النفسي الأمريكي

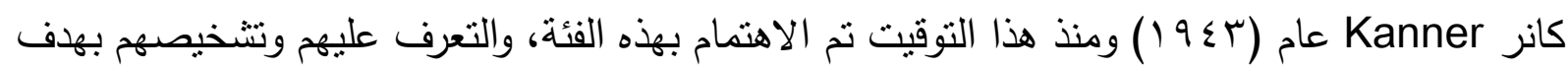
تحسين الخدمات المقدمة لهم وتقديمها في سن مبكر . ويعتبر نتخيص اضطراب الذاتوية من أكبر الشكلات التي نواجه المختصين في المجال، وتعود هذه

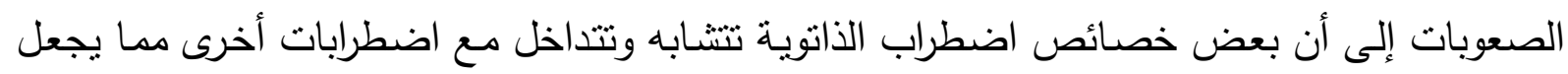

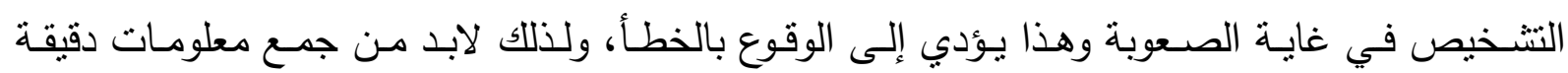
وصحيحة لتمييز الأفراد ذوي اضطراب الذانوية عن غيرهم من الاضطرابات الأخرى. مشكلة الدراسة: من خلال عملي مع الأطفال ذوي اضطراب الذاتوية لاحظت اختلافات واضحة في نتخيص اضطراب الذاتوية مما يؤثر على دقة التشخيص ودقة تقديم الخدمات والبرامج المتخصصة لهم وتتحدد مشكلة الدراسة الحالية في الأسئلة التالية: - ما - مالفروق في تشخيص اضطراب الذاتوية بين الدليل الرابع والدليل الخامس للاضطرابات العقلية؟ 


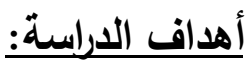

- التعرف على الفروق بين الدليل الرابع والدليل الخامس في تنخيص اضطراب الذانوية.

أهمبة الدراسة:

يمكن الإشارة إلى الأهمية النظرية والتطبيقية فيما يلي:

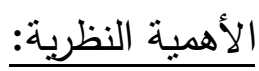

- إثراء تنظير عن تشخيص اضطراب الذاتويـة والدليل الرابع والدليل الخامس للاضطرابات

العقلية

- - التعرف على الفروق بين الدليل الرابع والدليل الخامس في تتخيص اضطراب الذاتوية.

الأهمية التطبيقية:

- تزويد المسئولين والمختصين بتتخيص اضطراب الذاتوية بالفروق بين الدليل الرابع والدليل

الخامس للاضطرابات العقلية على تتخيص اضطراب الذاتوية.

- - تحسين خدمات التشخيص للأطفال ذوي اضطراب الذاتوية.

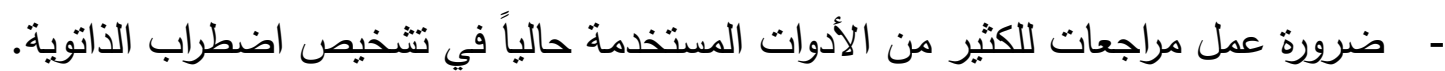

\section{مصطحات الارلسة:}

تشخيص:

تصنيف الأفراد على أساس الاضطراب أو الثذوذ أو مجموعة من الخصائص.

اضطراب الذاتوية: - n

اضـطراب عصـبي نــائي يـؤثر على مهـارات التواصـل والتقاعل الاجتمـاعي، ومصــاحب

بالسلوكيات النمطية ومحدودية الأنشطة والاهتمامات، يظهر خلال مرحلة الطفولة المبكرة ويؤثر على أداء الفرد للمهام اليومية.

الاليل النتخيصى والإحصائي الرابع للاضطرابات العقلية:

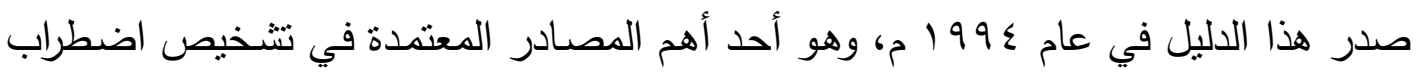

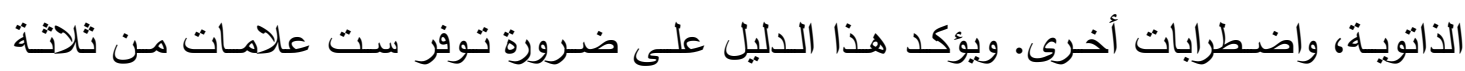
مجالات عند تثخيص اضطرابات الذاتوية.

وهذه المجالات الثلاثة هي: التقاعل الاجتماعي-التواصل-السلوكيات النمطية. الاليل التشخيصى والإحصائي الخامس للاضطرابات العقلية:

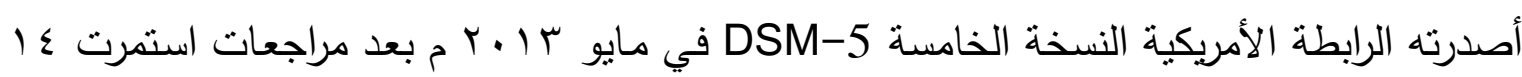

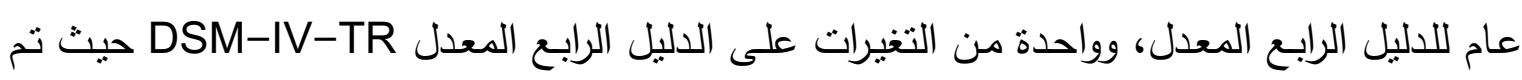


وضـع اضطراب أسبرجر واضطراب النمو الثـامل غير المحدد ضـمن مظلة اضطراب الذاتويـة، وأصبحت معاير التتخيص اثتين فقط هما (التواصل والتفاعل الاجتمـاعي - السلوكيات النمطية والاهتمامات والأنثطة المحدودة)، بحيث يجب أن ينطبق بنود التواصل والتفاعل الاجتماعي الثلاثة بالإضافة إلى بندين من أربعة بنود من المعيار الخاص بالسلوكيات النمطية والاهتمامات والأنشطة

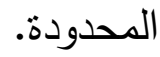

محددات الادراسة:

المحددات البشرية: ب ؟ فرد من ذوي اضطرابات النمو الثشاملة المحددات المكانية: محافظة القاهرة ومحافظة الثرقية ومحافظة الجيزة

\section{الإطار النظري والدراسـات السابقة}

اضطراب الأتوية

اضطراب الذاتوية هو في الأساس اضطراب سلوكي وهذا يعني وجود خلل في منظومة من الأنماط

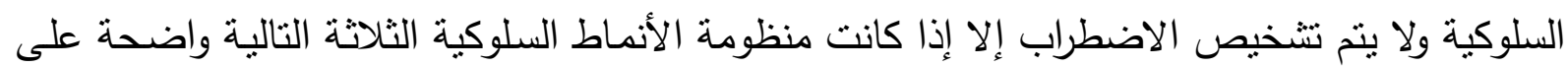
الطفل: - - خلل في علاقات الطفل بمحيطه الاجتماعي. - منثل الطفل في تطوير قدرات التواصل بشكل طبيعي. - اهتمامات الطفل ونشاطاته تكون محدودة ومكررة وليست واسعة وإبداعية. لابد من التأكد على أن التشخيص لا يتم لمجرد أن الطفل يعاني مشكلات تواصلية أو لأن لدى الطفل

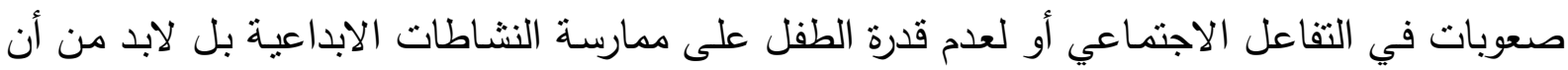
يُظهر الطفل قصوراً في كل الجوانب الثثلاثة مجتمعة.

(1) 1 : r.1. ، حافظ)

\section{تثخيص اضطراب الذاتوية:}

يعتبر التشخيص عملية أساسية تمكن من فهم الاضطراب وتشهيل عملية التخل في العلاج ومهم أن

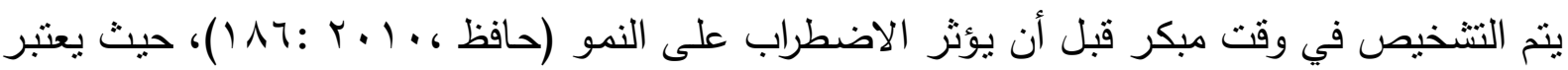

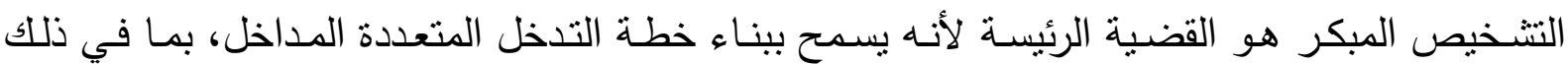
التعليم والتتخلات العلاجية التي تهدف إلى تخفيف الأعراض المختلفة.

(Rusu et al,2015:910) 
ويعتبر ليو كانر أول من وصف هذا الاضطراب في بداية الأربعينيات من القرن العشرين، وقد اعتمد

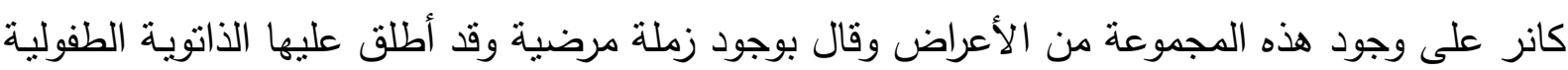

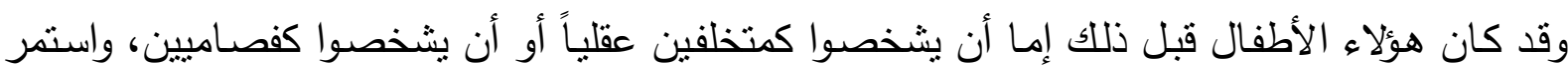
الخلط بين اضطراب الذاتويـة والفصـام حتى ظهرت الطبعة الثالثة من الدليل التتخيصـي والإحصـائي للأمراض العقلية (DSM-3) والتي حسمت الخلاف الدائر حول ارتباط التوحد بالفصام حيث اعتبر أن كلاهما كياناً مستقلاً.

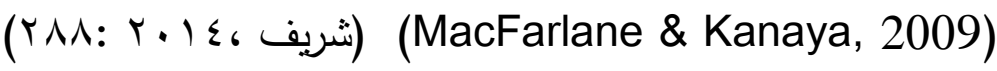

كما يعتبر تتخيص اضطراب الذاتويـة مشكلة شديدة التعقبد وذلك لصعوبة تميزهم عن غيرهم من الأطفال ذوب الإعاقة العقلية والأطفال الذين يعانون من اضطرابات في اللغة وترجع الصعوبة في ذلك

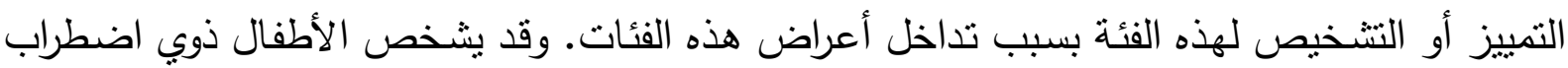

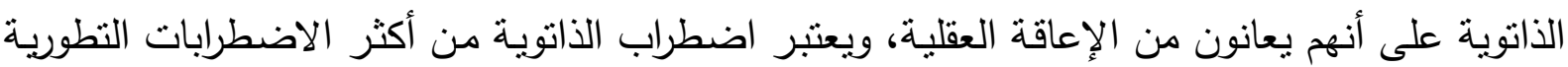
صعوبة ولا نوجد اختبارات طبية لنتخيص حالات اضطراب الذاتوية.

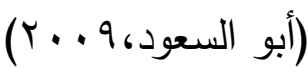

ويجب أن نشير إلى أنه يتوجب وجود عدد من الاختصاصين من ذوب الخبرات الجيدة في مجال

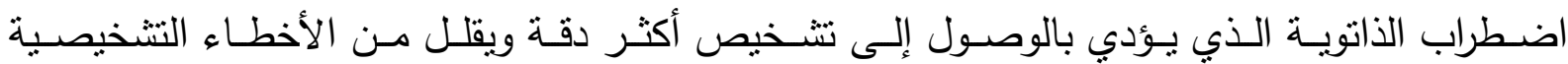

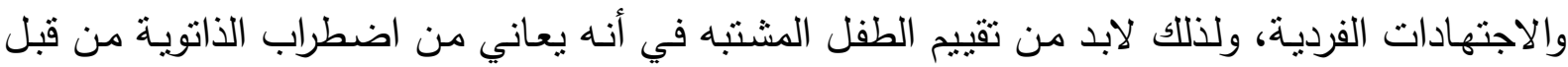
فريق يشمل العديد من النضصصات بحيث يقوم كل عضو فيه بحسب تخصصسه بتقييم الطفل ليتم في

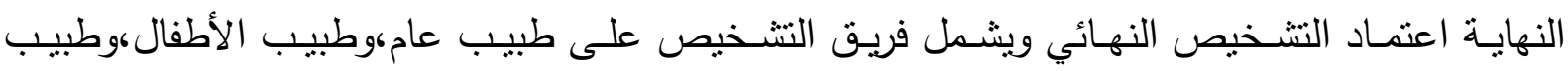

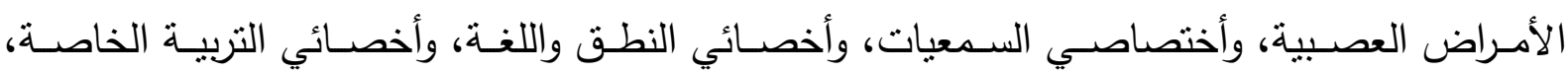
وأخصائي العلاج الطبيعي والوظيفي، والأخصائي الاجتماعي، والأخصائي النفسي ، والطبيب النفسي.

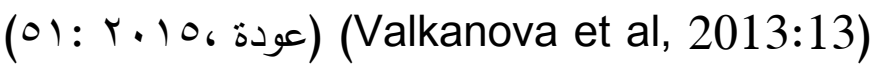

وترتكز عملية التقييم والتشخيص التي يقوم بها الفريق المتعدد التخصصات على عدة مبادئ من أهمها:

- - يجب أن تتم عملية التقييم للمجالات المتعددة في القدرات الوظيفية بما في ذلك المهارات التواصلية والسلوكية والتكيفية الوظيفية والذكائية. - - لابد من أن تعتمد عملية التقيم وتقسير نتائجها في ضدوء البعد النمائي العام، وتحديد جوانب القوة والضعف لدى الطفل. 
- أن يأخذ في الاعتبـار أن سلوك الطفل الذي يعاني مـن اضطراب الذاتويـة يتغير وفقاً للمواقف المختلفة ودرجة تنظيمها وتعقيدها. - تقييم مدى تأثنير اضطراب الذاتويـة على التفاعل الاجتماعي، باعتباره أحد أهم مـا يميز الطفل الذي يعاني من اضطراب الذاتوية. - - تقييم التكيف الوظيفي للطفل في سياق حياته اليومية والاستجابة لمنطلباتها.

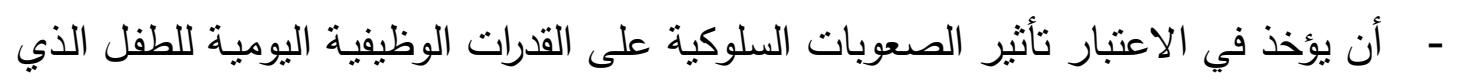
يعاني من اضطراب الذاتوية.

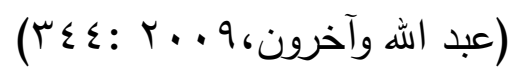

ولقد طورت بعض أدوات القياس المعينة بتشخيص الأطفال ذوي اضطراب الذاتوية، وهي عبارة عن

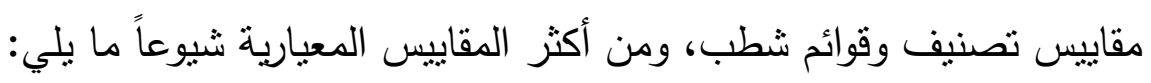
- - جدول الملاحظة التشخيصية لاضطراب الذاتوية (ADOS).

- المقابلة النتخيصية لاضطراب الذاتوية (ADI-R (Emberti et al,2016: 4) -

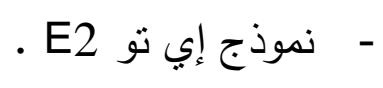

-

- مقياس تصنيف اضطراب الذاتوية في الطفولة (CARS).

- قائمة الثطب للأطفال ذوي اضطراب الذاتوية في سن المشي (CHAT). - مقياس جيليام لتصنيف اضطراب الذاتوية (Gilliam) -

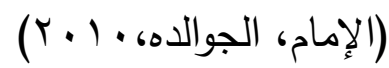
ويمكن حصر أهم هذه الأدوات المستخدمة في تشخيص اضطراب الذاتوية كما يلي:

\begin{tabular}{|c|c|c|}
\hline 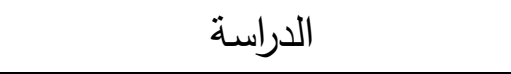 & اسم أداة التشخيص & 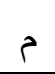 \\
\hline (Mattila et al,2011) & ADOS \& ADI-R & 1 \\
\hline (Gibbs et al,2012) & ADOS \& ADI-R & r \\
\hline (Matson et al,2012 a) & DSM-IV-TR/ICD Checklist & 0 \\
\hline (Matson et al,2012 b) & M-CHAT, BDI-2 & 7 \\
\hline (Worley \& Matson,2012) & DSM-IV-TR/ICD Checklist & 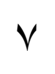 \\
\hline (McPartland et al,2012) & DSM-IV-TR/ICD Checklist & $\wedge$ \\
\hline (Neal et al,2012) & DSM-IV-TR/ICD Checklist & 9 \\
\hline (Geier et al ,2012) & CARS & 1. \\
\hline
\end{tabular}




\begin{tabular}{|c|c|c|}
\hline 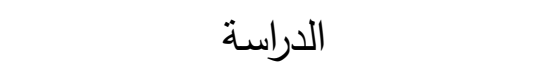 & اسم أداة التشخيص & s \\
\hline (Rieske et al,2013) & DSM-IV-TR/ICD Checklist & 11 \\
\hline (Barton et al,2013 ) & ADOS \& ADI-R & it \\
\hline (Mayes et al,2013) & CASD & r \\
\hline (Mazefsky et al,2013) & ADOS \& ADI-R & $1 \leq$ \\
\hline (Turygin et al,2013) & ADOS \& ADI-R & 10 \\
\hline (Beighley et al,2014) & DSM-IV-TR/ICD Checklist & 17 \\
\hline (Beighley \& Matson,2014) & DSM-IV-TR/ICD Checklist & iv \\
\hline (Beighley et al,2014) & DSM-IV-TR/ICD Checklist & 11 \\
\hline (Hiller et al,2014) & ADOS, ADI-R, ADEC, CARS & 19 \\
\hline (Kim et al,2014) & ADOS \& ADI-R & r. \\
\hline (Maenner et al,2014) & Clinical observations & r) \\
\hline (Mayes et al,2014) & Clinical observations DSM-5 & rr \\
\hline (Taheri et al,2014) & DSM-5 checklist & r \\
\hline (Young and Rodi,2014) & Clinical observations, ADI, CARS & $r \varepsilon$ \\
\hline (George et al ,2014) & CARS & ro \\
\hline (Yong-Hwee et al,2014) & CARS & ru \\
\hline (Malhi \& Singhi,2015) & CARS & rV \\
\hline (Chakraborty et al ,2015) & CARS & rᄉ \\
\hline (Garg et al ,2015) & ADOS-2 & rq \\
\hline (Anderson et al ,2015) & ADOS \& ADI-R & r. \\
\hline (Wiggins et al ,2015) & ADOS \& ADI-R & r \\
\hline (Emberti et al,2016) & ADOS & Tr \\
\hline
\end{tabular}

ويتضح من خلال العرض السابق أن أكثر الأدوات استخداما في تتخيص اضطراب الذانوية كلا من (ADderson et al وهذا يتفق مع دراسة أندرسون وآخرون (3117: 2015) (ADOS \& ADI-R)

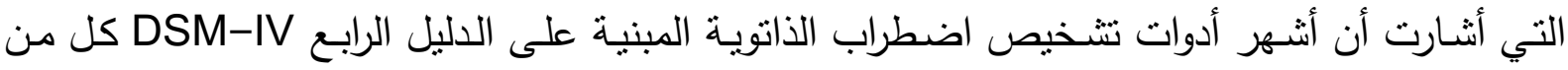


معايير نتخخيص اضطراب الذاتوية، كما نص عليها في الإصدار الرابع من الاليل التتخيصى

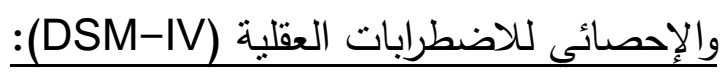

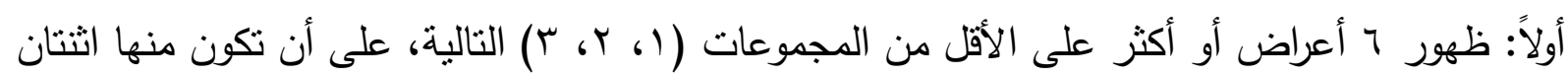
من أعراض المجموعة (1) على الأقل، بالإضافة إلى عرض الع ماحدة على الأقل من كل من المجموعتين

مجموعة (1) مجون

قصور كيفى Qualitative Impairment في قدرات التفاعل الاجتماعي . أ- قصور واضح فى استخدام صور متعددة من النواصل غير اللفظى، مثل تلاقى العيون أو تعابير الوجه أو حركات الجسم فى المواقف الاجتماعية والتواصل مع الآخرين. ب- الفشل فى تكوين علاقات مع الأقران تتتاسب مع العمر أو مرحلة النمو . ج-قصور القدرة على المشـاركة مـع الآخرين فى الأنشطة الترفيهيـة أو الهوايـات أو إنجاز أعمـال مشتركة معهم. د-غياب المشاركة الوجدانية أو الإنفعالية أو التعبير عن المشاعر.

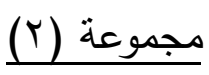

قصور كيفي في القدرة على التواصل Qualitative Communication Impairment كما يكثفها واحد على الأقل من الأعراض الآتية: أ-تأخر وغياب تام في نمو القدرة على التواصل بالكلام (التخاطب) وحده (بدون مساندة أى نوع من الأن أنواع التواصل غير اللفظى للتعويض عن قصور اللغة).

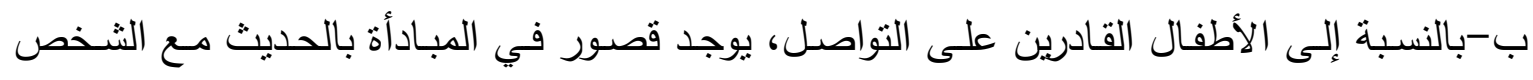

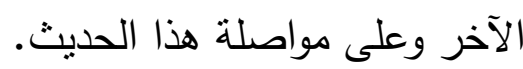

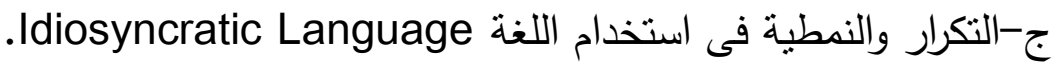
د-غياب القدرة على المشاركة فى اللعب الإيهامى أو النقليد الاجتماعي الذي يتتاسب مـع العمر

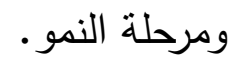

مجموعة (r)

اقتصار أنشطة الطفل على عدد محدود من السلوكيات النمطية كما يكثف عنها واحد على الأقل من الأعراض الآتية: - الأن أ-استغراق أو اندماج كلى فى واحد أو أكثر من الأنشطة أو الاهتمامات النمطية الثناذة من حيث طبيعتها أو شدتها. 
ب-الجمود وعدم المرونـة الواضـح فى الالتزام والالتصـاق بسلوكيات وأنشطة روتينية أو طقوس لا جدوى منها.

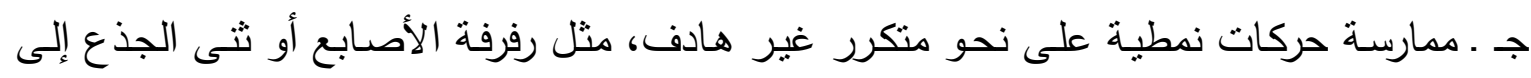
الأمام والخلف أو تحريك الأذرع أو الأيدى أو قفز بالأقدام... إلخ. د-انشغال طويل المدى بأجزاء أو أدوات أو أجسام مع استمرار اللعب بها لها لمدة طويلة، مثل سلسلة

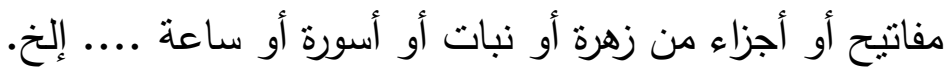
ثانياً: تأخر أو شذوذ وظيفى يكون قد بدأ فى الظهور قبل العام الثالث من عمر الطفل فى واحد مما يلي:

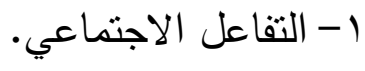
r - استخدام اللغة فى التواصل أو في التخاطب. r- اللعب الرمزى أو الإيهامى التخيلي (أخذ أدوار الكبار). ثالثاً: غياب أعراض متلازمة الريت Rett أو اضطراب الطفولة التفكي. التهي.

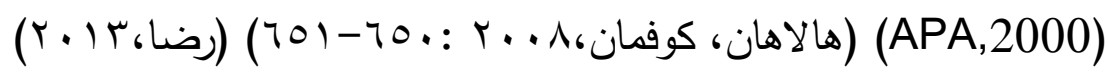
تاريخ الدليل النتخيصى والإحصائي الخامس DSM-5 للاضطرابات العقلية: في عام 999 ام خططت الرابطة الأمريكية للطب النفسي لمراجعة الدليل الرابع DSM-IV، ونظمت لهنية

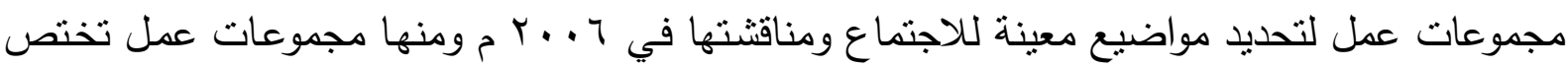
بالاضطرابات النمائية العصبية التي ركزت على معايير تثخيص اضطراب الذاتوية، وهذه المجموعة بحث التعديلات والتغيرات المطلوبة تعديلها في تثخيص اضطراب الذانوية (Wodrich et al:

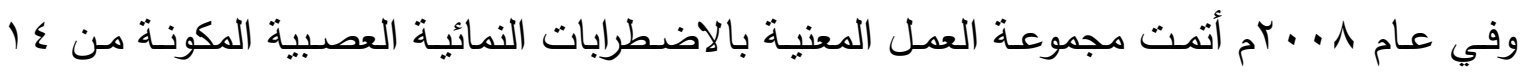
عضو مراجعة النسخة الحالية من الدليل التتخيصـي والتصنيفي الخامس DSM-5، بحيث أصبحت مظلة اضطراب الذاتوية تتمل (اضطراب الذاتوية التقليدي-اضطراب أسبرجر - اضطراب النمو الثشامل

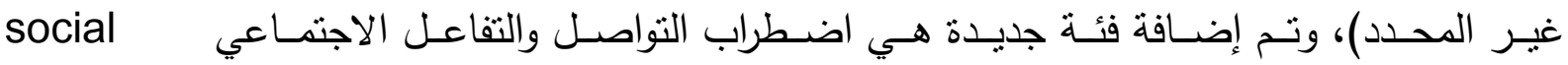
communication disorder (SCD) (King et al, 2014) (King et al., 2013) ونت عمل نقاشات واجتماعات حول الموافقة أوعدم الموافقة على هذه التعديلات من خلال المختصين والمراكز البحثية وقدمت الرابطة الأمريكية للطب النفسي هذه التعديلات في ديسمبر بات الـ بم وتم اعتمادها

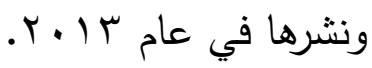

(Grant\& Nozyce, 2013) 
واعتمدت الرابطة الأمريكية للطب النفسي علي أدلة واضـحة في تطوير وتعديل النسخة الجديدة وتغيرت المظلـة من الاضطرابات النمائية الثـاملة إلي اضطراب الذاتويـة وتم دمـج معيار التواصل مـع معيار التفاعل الاجتماعي في معيار واحد فقط أكثر إجمالاً تحت مسمى التواصل والتفاعل الاجتماعي (المعيار الأول)،ويحتوي هذا المعيار علي ثنلاثة بنود يجب أن ينطبقوا جميعا على الطفل المشخص باضطراب الذاتويـة، وأيضـاً يجب أن ينطبق على الأقل بندين من البنود الأربعة مـن (المعيار الثاني) الخاص بالسلوكيات النمطية والمتكررة والاهنمامات والأنشطة المحدودة. (Matson et al, 2012) كما يجب تحديد درجة المساندة أو الدعم المطلوب لكل حالة وفقاً لمستوى شدة الأعراض أو مستوى الخطورة لديها في مجال التواصـل والتفاعل الاجتمـاعي من جهة ومجال السلوكيات النمطيـة والمتكررة والاهتمامات والأنشطة المحدودة من جهة أخرى، حيث تم تقسيم الاضطراب إلى ثلاثة فئات تختلف في درجة الدعم والمسـاندة (المستوى " (" يتطلب دعم-المستوى "ب" يتطلب دعم كبير -المستوى "ب" يتطلب دعم كبير جدا).

( Weitlauf et al, 2014)

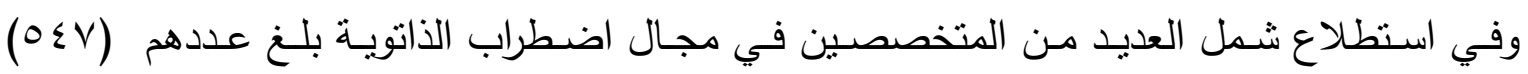
متخص لدراسة التغيرات الجديدة في الدليل الخامس ومدى موافقتهم عليها، أثنارت النتائج إلى أن ع,بـو مـازالوا يقتتعـون أن هنـاك اختلافـات بـين اضـطراب الذاتويـة واضـطراب أسبرجر ،كمـا وجدوا أن نسبة

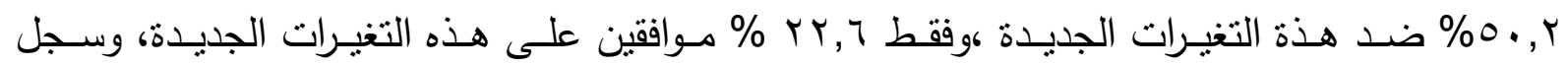
المعترضـين على التغيـرات الجديـدة العديـد مـن الملاحظـات حـول الاختلافـات بـين اضطراب اسـبرجر وحـالات الذاتويـة مرتفعـة الأداء وبين الأنـواع المختلفـة مـن الاضـطراب واختناف الاحتياجـات والـدعم والمساندة في كل نوع.

(Kite et al, 2013)

ويتفق الباحث مع ما توصلت إليه دراسة (2799: 2014, Bennett et al) ، من حيث مراعاة بنـود الدليل التشخيصـي والتصنيفي الخـامس DSM-5 عند تشـخيص الأطفال ذوي اضطراب الذاتويـة ومراجعة بعض المقاييس والأدوات المستخدمة حالياً في تشخيص اضطراب الذاتوية المبنية على الدليل الرابع DSM-IV منل ( ADI-R\&ADOS ). التغييرات المواكبة للاليل التشخيصي الخامس DSM-5 وأثنارها على تشخيص اضطراب الذاتوية: ولقد واكب نشر النسخة الخامسة DSM-5 جدلاً واسعاً منذ الإعلان عن التغييرات الجديدة في معايير التشخيص التي اقترحها الجمعية الأمريكية للطب النفسي (APA, 2013)، وزادت التساؤلات حول صحة تشخيص DSM-5، حيث توجد اختلافات بين الدليلين الرابع والخامس في تشخيص اضطراب الذاتوبة 
في العديد من الدراسات منها دراسة (Mattila et al, 2011)، ودراسة(Wilson et al, 2013) ،

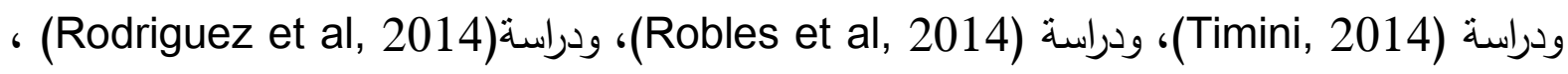

ودراسة (Romeroa, 2016:268).

وواحدة من الخلافات المهمة في النسخة الخامسة هي تثخيص اضطراب الذاتوية كما نصت عليه

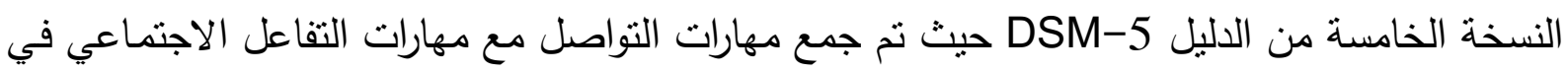

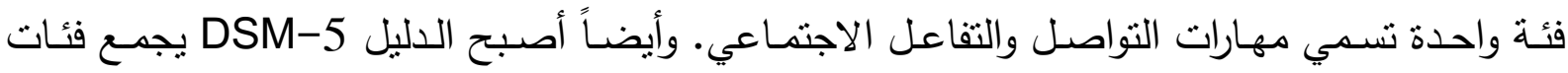
مختلفة من الاضطرابات النمائية الثشاملة (PDD) تحت مظلة واحدة تسمي اضطراب الذاتوية مع تحديد مستويات الخطورة وخدمات الدعم والمساندة المختلفة.

(McPartland et al, 1012), (Worley\& Matson, 2012)

ومن جهة أخرى هناك اتفاق على مختلف المواد في الدليل الخامس، كما يتضمن التصنيف الجديد

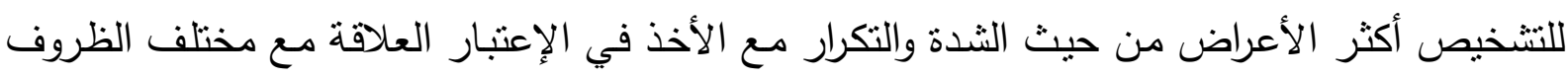

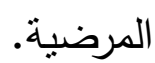

( Gibbs et al, 2012)

كمـا تتـــابه أعرض كل من اضطراب الذاتويـة الكلاسيكي واضطراب اسبرجر واضطراب النمو الثنامل غير المحدد، وهذه أعراض تختلف من البسيط إلى الثديد في الدعم والمساندة في مجالين هما

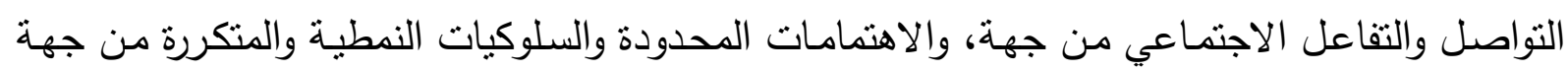

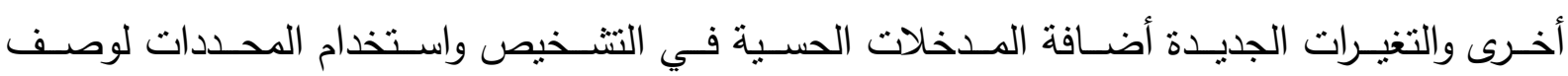
الاضطرابات المصاحبة وشدة الاضطراب أو عوامل الخطورة وهذه الإضافات لم تكون موجودة في الدليل

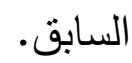

(Prykanowski et al, 2015:34)

ومن جهة أخرى لم يتاح استخدام الدليل التشخيصي الخامس DSM-5 حتى الأن في إيطاليا ويتم

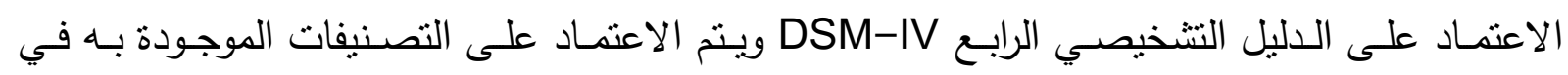
تتخيص وتصنيف الاضطرابات النمائية الثناملة.

(Emberti et al, 2016: 3)

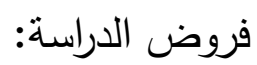
- - توجد فروق بين الدليل النتخيصي والتصنيفي الرابع والدليل النتخيصي والتصنيفي الخامس في تشخيص اضطراب الذاتوية. 
- - توجد فروق بين الدليل التشخيصي والتصنيفي الرابع والدليل التشخيصي والتصنيفي الخامس في تنخيص اضطراب الذانوية بين الذكور والإناث.

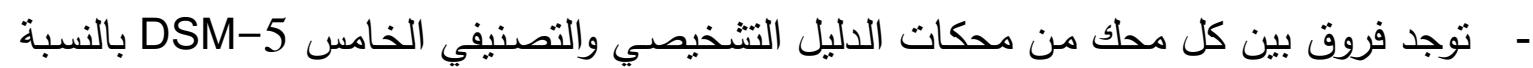
للأفراد من ذوي اضطرابات النمو الثاملة.

إجراءات الدراسة:

أولاً منهج الدراسةة:

استخدم الباحث المنهج الوصفي في هذه الدراسة للتعرف على الفروق بين الدليل التتخيصي والتصنيفي الرابع DSM-4 والدليل التشخيصي والتصنيفي الخامس DSM-5 في تتخيص اضطراب الذاتوية. ثانياً عينة الدراسة: يمكن وصف عينة الدراسة كما يلي:

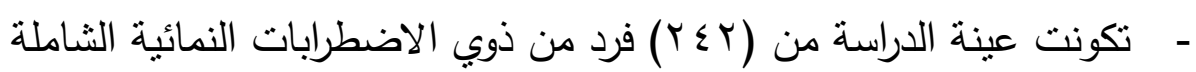

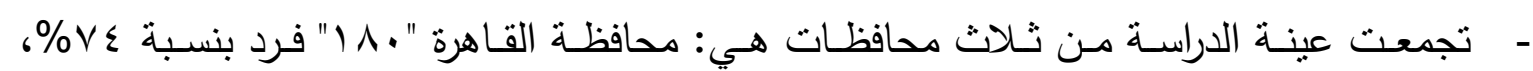

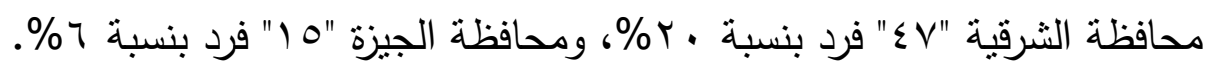

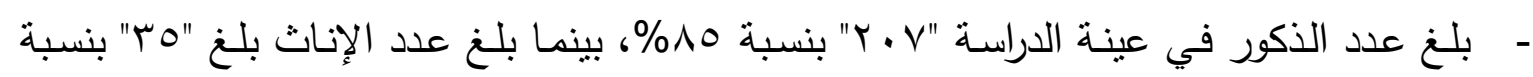
$\% 10$

\section{أدوات الارراسة:}

(APA,2013) DSM-5 الاليل التشخيصي والتصنيفي الخامس للاضطرايات العقلية قام الباحث بتطبيق المحكات التتخيصية للاليل التشخيصي والتصنيفي الخامس DSM-5 على لى لى

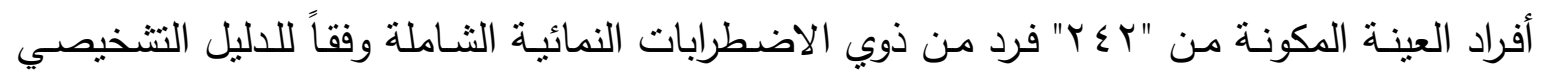

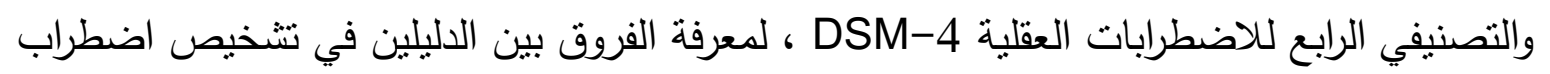
الذانوية وتتمنل هذه المحكات فيما يلي:

المعالم التتخيصية للاليل التشخيصى والتصنيفى الخامس DSM-4 A عجز ثابت في التواصل والتفاعل الاجنماعي في سياقات متعددة، في الفترة الراهنة أو كما ثبت عن طريق التاريخ وذلك من خلال ما يلي، (الأمتلة توضيحية، وليست شاملة:)

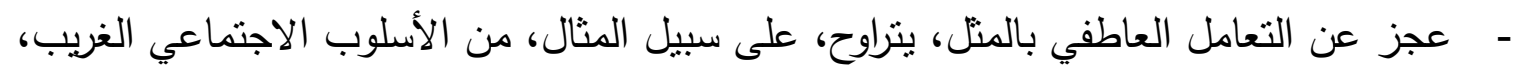

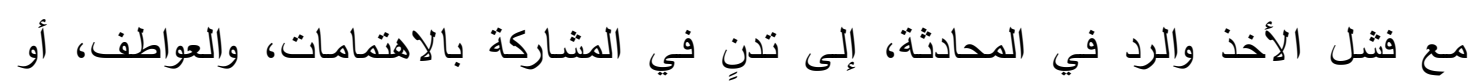
الانفعالات، يمتد إلى عدم البدء أو الرد على التفاعلات الاجتماعية. 
- العجز في سلوكيات التواصل غير اللفظية المستخدمة في التفاعل الاجتماعي، يتراوح من ضعف تكامل التواصل اللفظي وغير اللفظي، إلى الثذوذ في التواصل البصري ولغة الجسد أو العجز

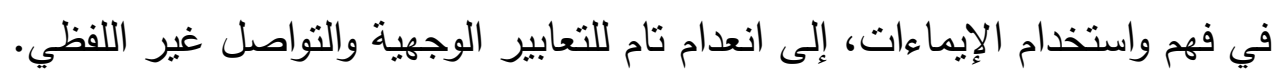

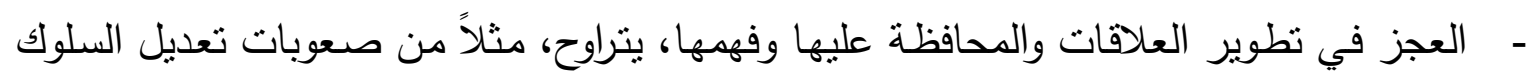
لتلائم السياقات الاجتماعية المختلفة، إلى صعوبات في مشاركة اللعب التخيلي أو في تكوين صداقات، إلى انعدام الاهتمام بالأقران. تحديد الثدة الحالية:

تستند الثدة على ضعف التواصل الاجتماعي وأنماط السلوك المحددة، المتكررة.

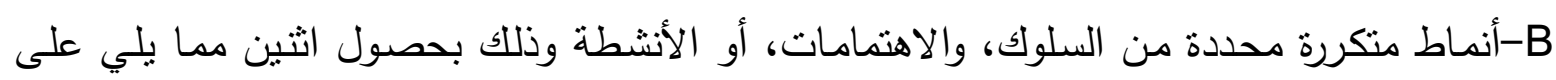

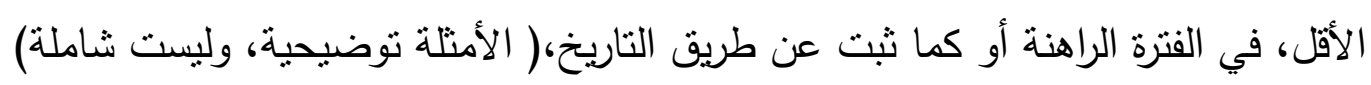

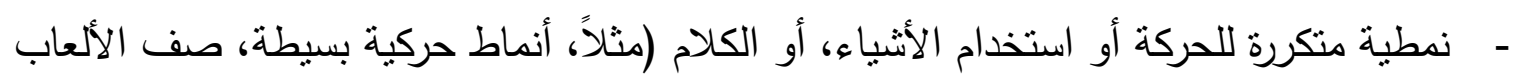

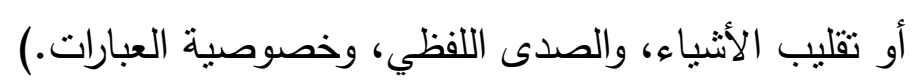

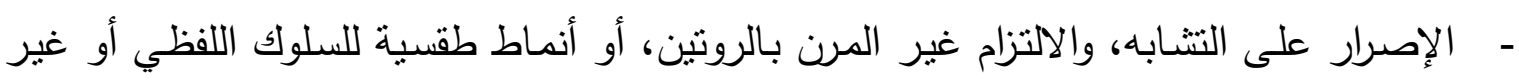

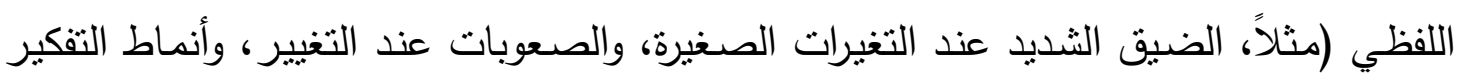
الجامدة وطقوس التحية، والحاجة إلى سلوك نفس الطريق أو تتاول نفس الطعام كل يوم.)

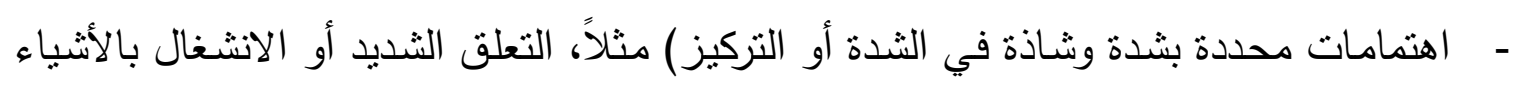

غير المعتادة، اهتمامات محصورة بشدة مفرطة المواظبة.) -

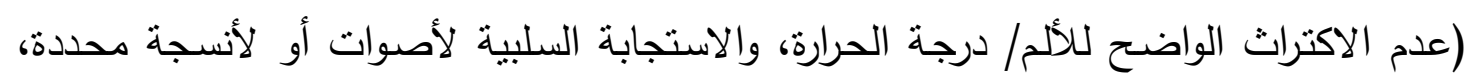

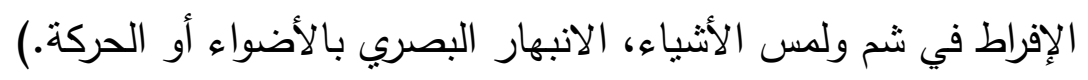
تحديد الثدة الحالية:

تستند الثدة على ضعف التواصل الاجتماعي وأنماط السلوك المحددة، المتكررة. -C تظهر الأعراض في فترة مبكرة من النمو (ولكن قد لا يتوضح العجز حتى تتجاوز متطلبات التواصل الاجتماعي القدرات المحدودة أو قد تحجب بالاستراتيجيات الكُتعلمة لاحقاً في الحياة.)

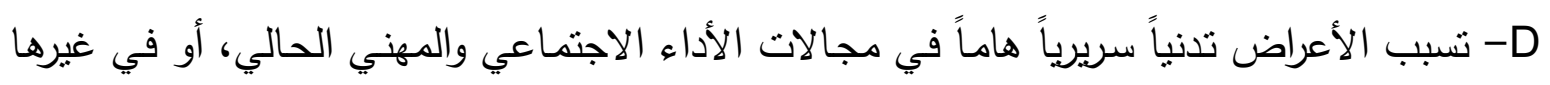

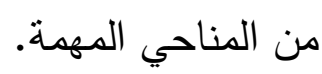
لا تُفر هذه الاضطرابات بشكلٍ أفضل بالإعاقة العقلية (اضطراب النمو الذهني) أو تأخر النمو

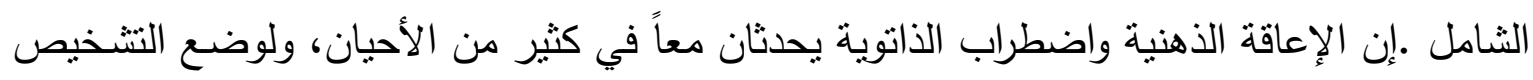


المرضي المشترك للإعاقة الذهنية واضطراب الذاتوية، ينبغي أن يكون التواصل الاجتماعي دون المتوقع للمستوى التطوري العام.

نتائج الدراسة وتفسبرها نتائجج الفرض الأول:

يــص الفـرض الأول علـي أنـه "توجد فـروق بـين الـليل النشخيصـي والتصـنيفي الرابـع والـليل التشخيصي والتصنيفي الخامس في تشخيص اضطراب الذاتوية". وللتحقق من صحة هذا الفرض، تمت مقارنة مدى انطباق المحكات النشخيصية للدليل التشخيصي والتصـنيفي الخـامس DSM-5 على عينـة الدراسـة المكونـة مـن "بـ ؟" فرد مـن ذوي اضطرابات النمـ

الشاملة وكانت النتائج كما هي موضحة بالجدول (1)

\section{جدول (1)}

الفروق بين الدليل التشخيصي والتصنيفي الرابع والدليل التشخيصي والتصنيفي الخامس في تشخيص اضطراب الذاتوية

\begin{tabular}{|c|c|c|c|c|c|}
\hline نسبة المئوية للفروق & النسبة المئوية لانطباق & عدد الأفراد التي تنطبق & العدد & نوع الاضطراب & p \\
\hline$\% \varepsilon, 0$ & $\% 90,0$ & IV. & IVA & اضطراب الذاتوية التقليدي & 1 \\
\hline$\% \circ r, v$ & $\% \leq V, r$ & 9 & 19 & اضطراب أسبرجر & r \\
\hline$\% \quad r \varepsilon$ & $\% \vee 4$ & rr & $\varepsilon r$ & اضطراب النمو الشامل غير & $r$ \\
\hline$\%$ & $\% 1 \ldots$ & $r$ & $r$ & اضطراب رت & $\varepsilon$ \\
\hline
\end{tabular}




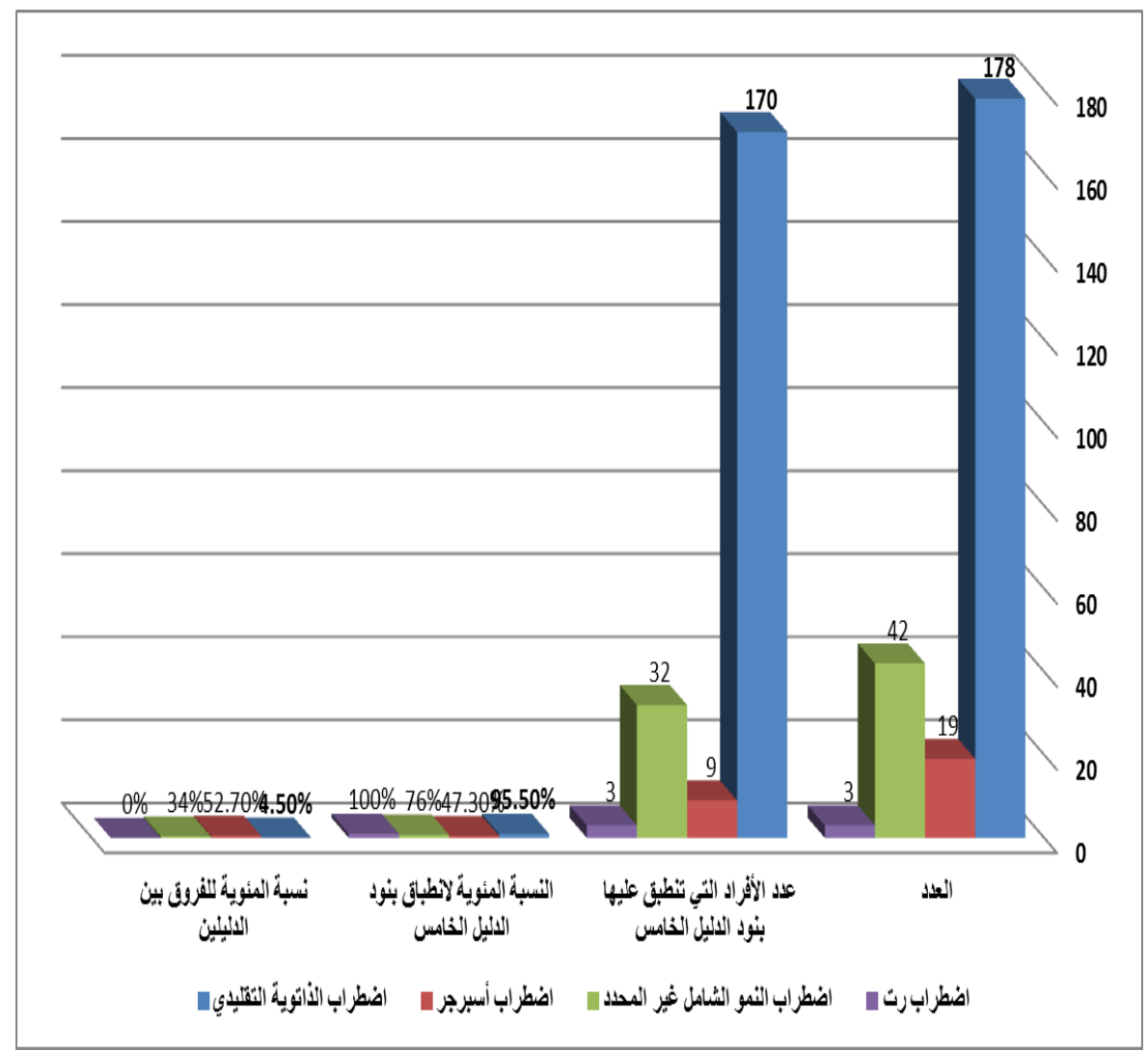

شكل (1) يوضح الفروق بين الدليل التتخيصي والتصنيفي الرابع والدليل التشخيصي والتصنيفي الخامس في تنخيص اضطراب الذانوية

\section{نتائج الفرض الثاني:}

ينص الفرض الثناني علي أنسه نوجد فروق بـين الدليل النتخيصـي والتصـنيفي الرابـع والدليل التتخيصي والتصنيفي الخامس في تتخيص اضطراب الذاتوية بين الذكور والإناث. وللتحقق من صحة هذا الفرض، تمت مقارنة مدى انطباق المحكات التثخيصية للدليل النتخيصي والتصنيفي الخامس DSM-5 على عينة الدراسة المكونة من "rع ؟" من ذوب اضطرابات النمو الثناملة وفق الدليل التشخيصي والتصنيفي الرابع DSM-4 وكانت النتائج كما هي موضحة بالجدول (r)

\section{جدول (r)}

الفروق بين الدليل التشخيصي والتصنيفي الرابع والدليل التشخيصي والتصنيفي الخامس في تشخيص اضطراب الذاتوية بالنسبة للنوع

\begin{tabular}{|c|c|c|c|c|c|}
\hline نلفروق بين الدليلين & 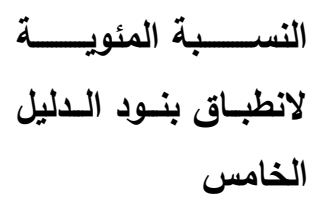 & 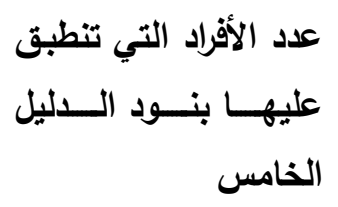 & | العدد & |النوع & م \\
\hline$\%$ & $\% \wedge \vee$ & $1 \wedge$ & $r \cdot v$ & الذكور & 1 \\
\hline$\% r$ & $\% q \vee$ & $\Gamma \varepsilon$ & ro & الإناث & $r$ \\
\hline
\end{tabular}




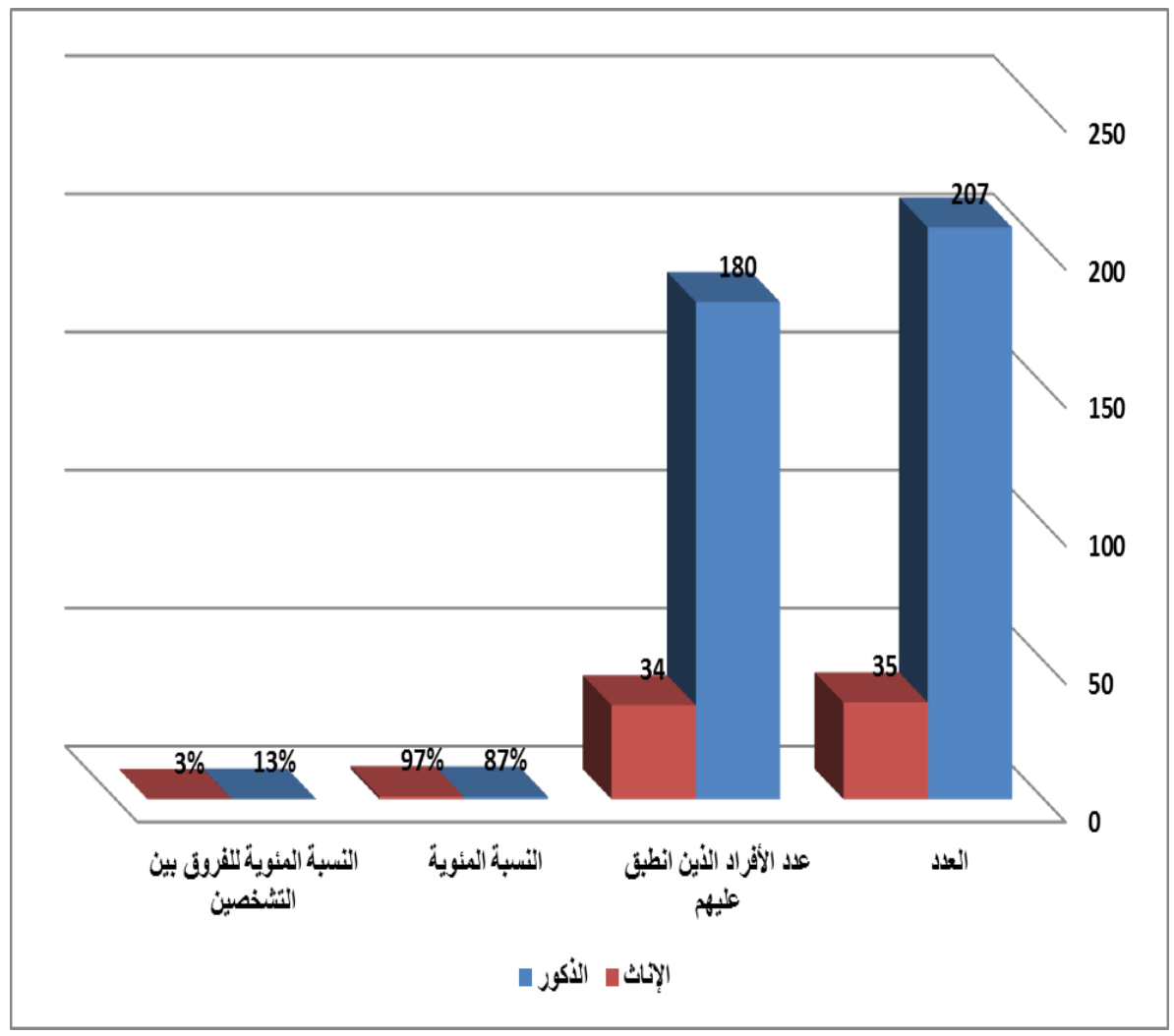

جدول (r) الفروق بين الدليل التشخيصي والتصنيفي الرابع والدليل التشخيصي والتصنيفي الخامس في تشخيص اضطراب الذاتوية بالنسبة للنوع ولنئ

نتائج الفرض الثالث:

ينص الفرض الثالث علي أنه توجد فروق بين كل محك من محكات الدليل التتخيصي والتصنيفي الخامس DSM-5 بالنسبة للأفراد من ذوي اضطرابات النمو الثاملة.

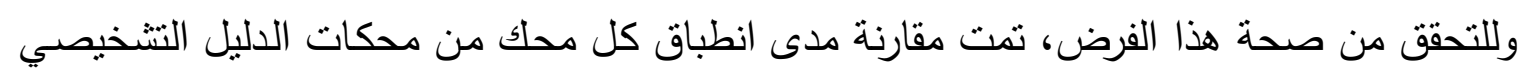
والتصنيفي الخامس DSM-5 على عينة الدراسة المكونة من "بع ب" من ذوي اضطرابات النمو الثناملة

وكانت النتائج كما هي موضحة بالجدول (r) 
جدول (r)

نسبة انطباق كل محك من محكات الدليل التتخيصي والتصنيفي الخامس DSM-5 على أفراد العينة المكونة من (r Y r) فرد ذوي اضطرابات النمو الثناملة

\begin{tabular}{|c|c|c|}
\hline 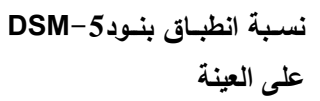 & بنود كل محك من محكات الدليل الخامس DSM-5 & 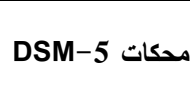 \\
\hline$\% 90, \wedge$ & صعوية في التبادل العاطفي وسلوكيات التواصل اللفظي المستخدمة في التفاعل الاجتماعي & \multirow{3}{*}{ A } \\
\hline$\% 90, \wedge$ & صعوية في سلوكيات التواصل غير اللفظي المستخدمة في التفاعل الاجتماعي & \\
\hline$\% 99,4$ & صعوية في إنثاء العلاقات أو الحفاظ عليها أو فهمها & \\
\hline$\% \wedge q, r$ & نمطية وتكرار في حركات الجسم أو استخدام الأثشياء أو الكلام & \multirow{4}{*}{ B } \\
\hline$\% \vee 1, \varepsilon$ & الإلصرار على المثليـة (تماثل الأفعال)، وارتبـاط دائم بالأفعال الروتينيـة، أو طقسية أو الطبيعة أو & \\
\hline$\% \wedge \bullet, 0$ & اهتمامات محددة وثثابتة بثكل كبير ويصورة غير طبيعية من ناحية الثدة والتركيز & \\
\hline$\% \wedge \cdot, \bullet$ & فرط أو انخفـاض حركي نتيجـة للمــخلات الحسية، أو اهتمامـات غير طبيعـة بالجوانب الحسية & \\
\hline$\% 9 \vee, 0$ & يجب أن تظهر الأعراض في الفترة المبكرة من نمو الطقل. & C \\
\hline$\% १ \wedge$ & يجب أن تسبب الأعراض ضراً واضحاً في المواقف الاجتماعية والوظيفية والمواقف الحياتيـة الأخرى & D \\
\hline$\% 9$. & النسبة العامة & \\
\hline
\end{tabular}

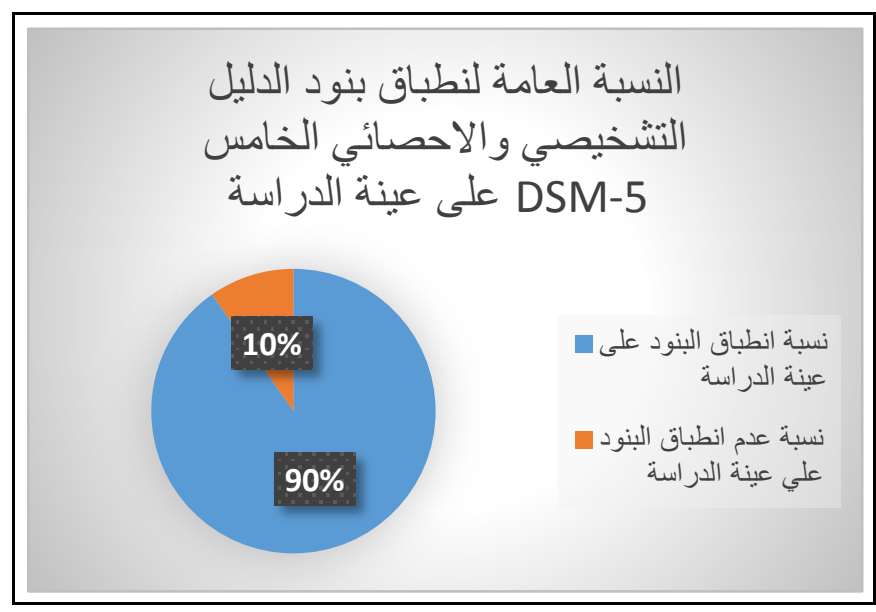

شكل (r) نسبة انطباق كل محك من محكات الدليل التتخيصي والتصنيفي الخامس DSM-5 على أفراد العينة المكونة من (r r r) فرد ذوبي اضطرابات النمو الثاملة

تتشير النتائج إلي وجد فروق فروق بين الدليل التشخيصي والتصنيفي الرابع والدليل التشخيصي والتصنيفي الخامس في تشخيص اضطراب الذاتوية الذي يشمل كل من (اضطراب الذاتوية التقليدي -

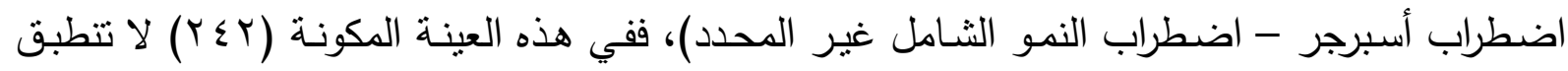
معايير اضطراب الذاتوية وفق الدليل التشخيصي الخامس DSM-5 على (• ( \% م) من الأفراد وبالتالي 
يتحقق الفرض، وتتفق هذه النتيجة مـع العديد من الدراسات التي أنشارت لوجد فروق بين الدليلين الرابع والخامس ويمكن توضيح ذلك فيما يلي:

ففي دراسة (Gibbs et al , 2012:1750) إلى مقارنة النتائج النتخيصية باستخدام معايير كلاً

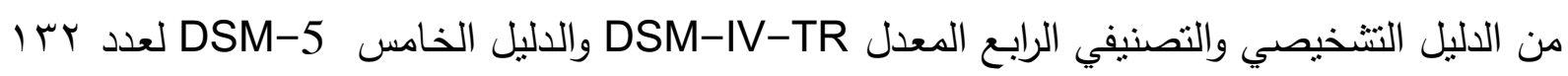
طفلا نم تثخيصهم باضطرابات النمو الثشاملة وفقاً للاليل الرابع المعدل ، ووجد اتفاق وعدم اختلاف في

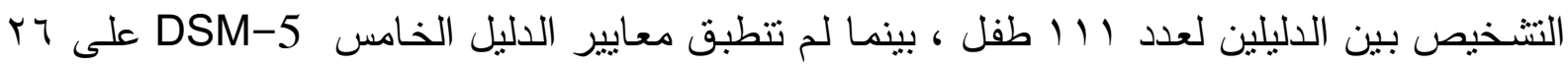

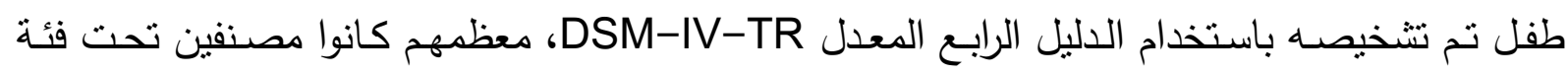

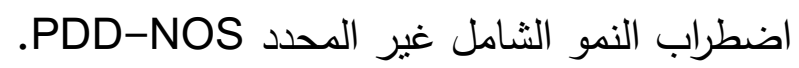
وفي دراسة (McPartland et al, 2012) بتحليل تتخيص عدد rra طفل شخصوا باضطرابات

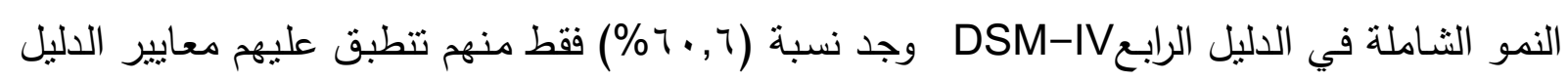

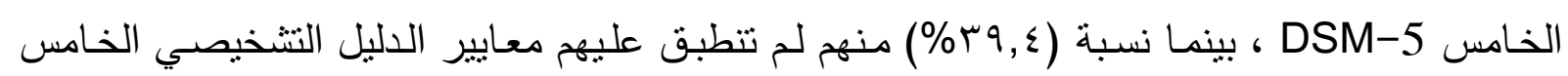

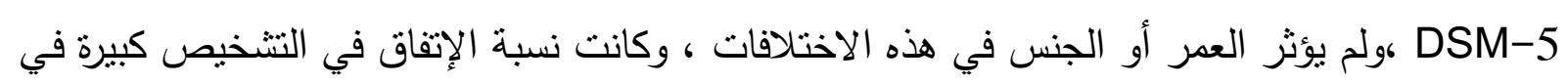

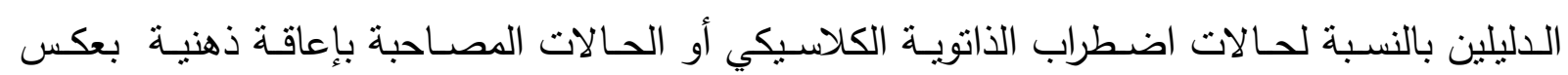
الحالات غير المصاحبة بإعاقة ذهنية وأيضا اضطراب أسبرجر واضطراب النمو الثامل غير المحدد.

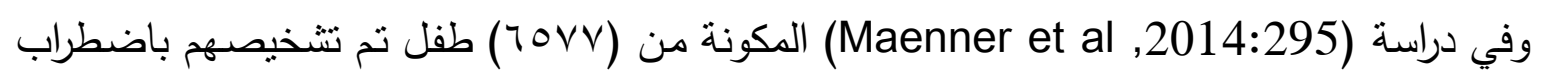

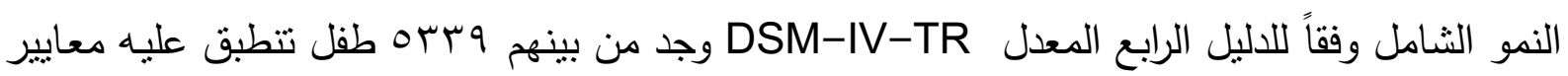

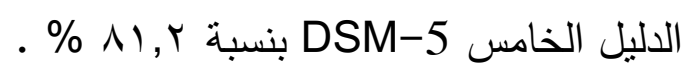
وفي دراسة (Young \& Rodi, 2014: 758)، التي تكونت من · (Y طفل من ذوي الاضطرابات

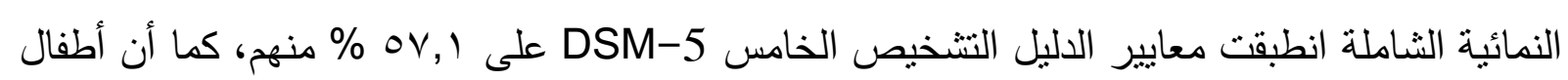
اضطراب أسبرجر واضطراب النمو الثشامل غير الدحد من أكثر الفئات التي لم تتطبق عليها معايير

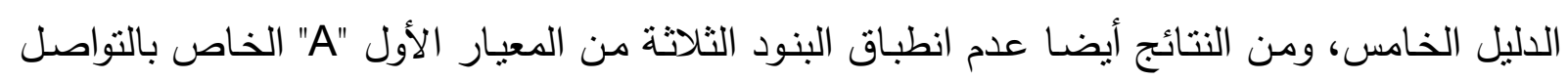

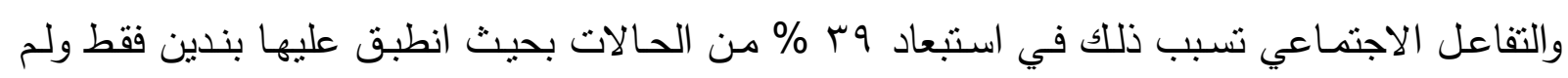
يشخصوا باضطراب الذاتوية. وفي دراسة (2753: Steensel et al, 2015 نكونت عينة الدراسة من (•9) طفل من ذوي

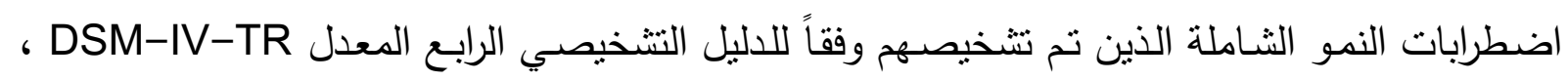

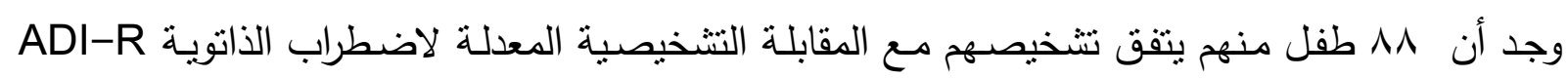

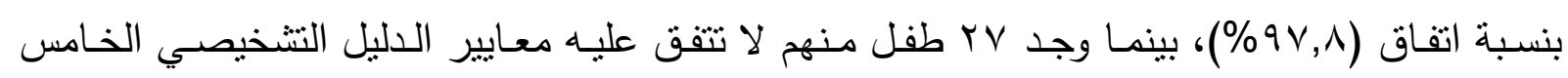

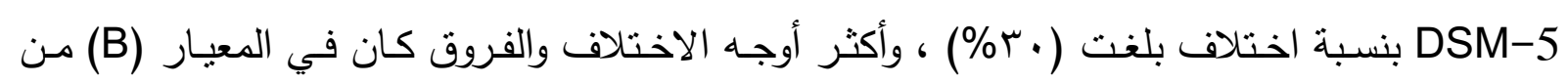


معايير الدليل التشخيصي الخامس DSM-5 الخاصة بأنماط المحدودة والمتكررة من السلوك والاهتمامات

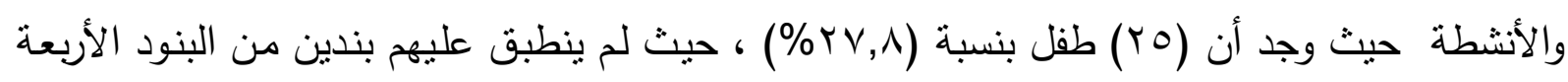
في هذا المعيار (B) وانطباق بندين شرط للتشخيص في المعيار الثاني، وهؤلاء الأطفال كانوا مشخصين

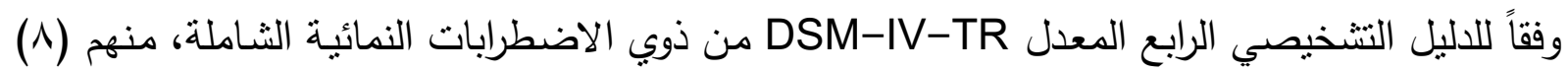

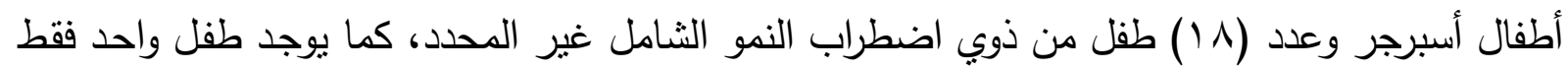

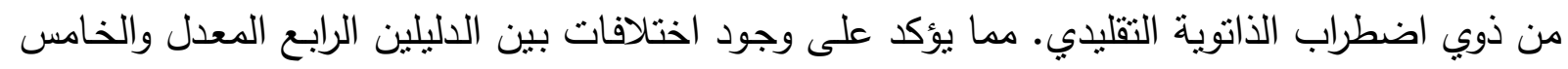
لتصنيف وتتخيص الاضطراب العقلية في تنخيص اضطراب الذاتوية.

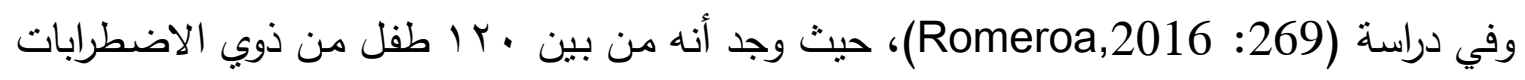

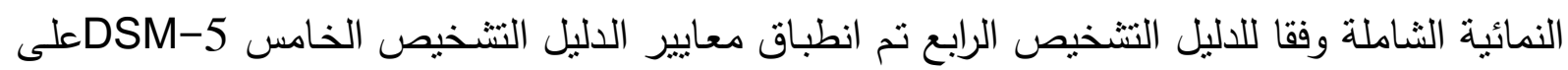

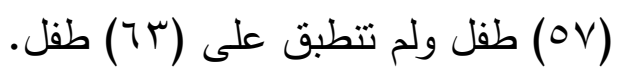
ولقد وجد الباحث أيضـا في الدراسـة الحالية أن أكثر الفئات التي لا تتطبق عليها معايير الدليل

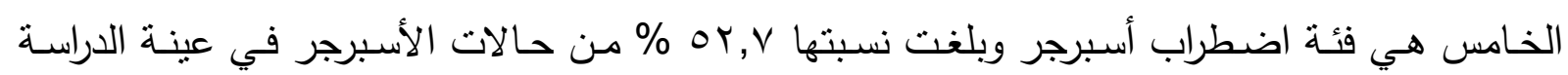
المكونة من "9 ا" فرد من ذوي اضطراب أسبرجر ، ويليه اضطراب النمو الثـامل غير المحدد حيث بلغت

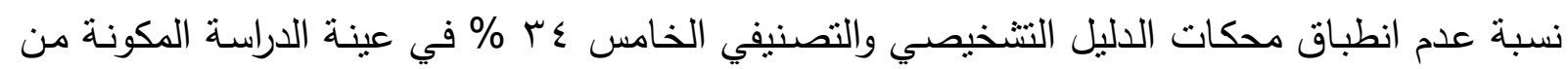

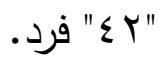

كمـا تبـدو العلاقـة عكسية بـين الحـالات المرتفعـة مـن ذوبي الاضـطرابات النمائيـة الثـاملة وبـين

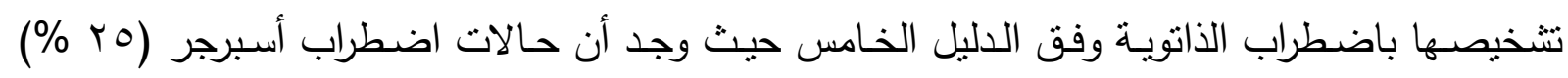

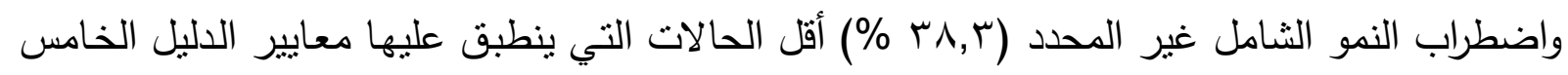

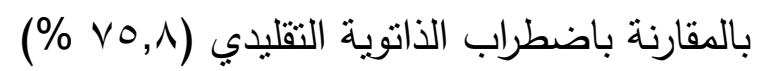
(Gibbs et al,2012) (McPartland et al ,2012) ومن نتائج الدراسة أيضا وجود فروق في انطباق معايير الدليل الخامس بين الجنسين، ففي الذكور

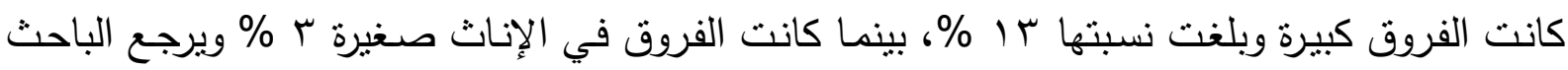

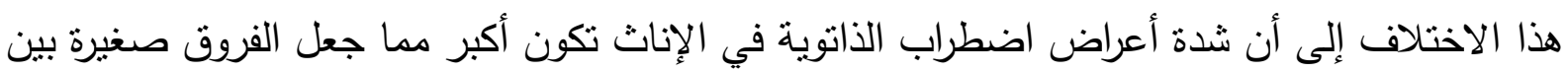
الدليل بالنسبة للإناث وكبيرة بالنسبة للذكور . وتتير النتائج لوجود فروق في نسبة انطباق كل محك من محكات الدليل التشخيصي والتصنيفي

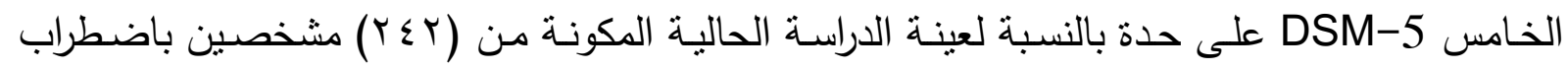

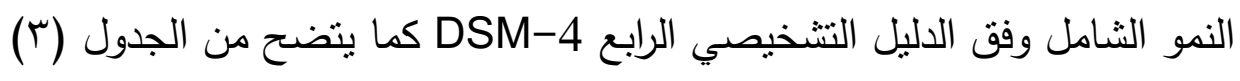


كما يشير الباحث إلى أن نسبة العامة للاتفاق بين الدليلين في الدراسة الحالية بلغت .9 \% و أعلى

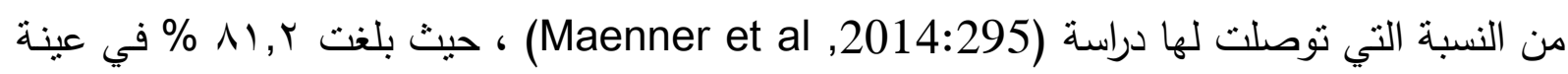

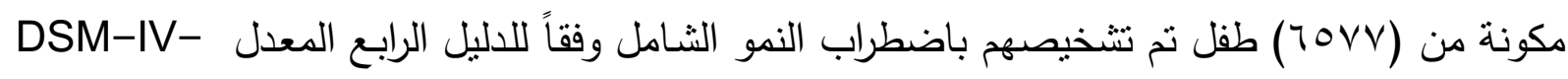

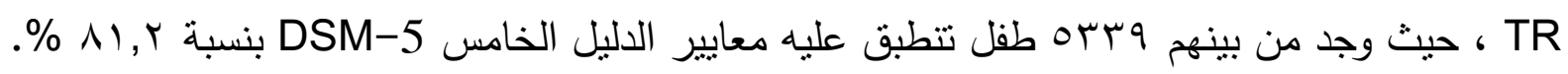

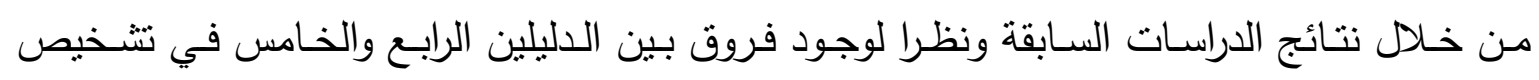
اضطراب الذانوية يجب تطوير المقاييس المستخدمة حالياً في تشخيص اضطراب الذانوية لتكون أكثر دقة لئن وموضوعة وصدق بحيث تسنوفي معايير اضطراب طيف الذاتوية الواردة في النسخة الخامسة من الدليل (WSM-5) وهذا يتفق مع دراسة (White et al, 2014).

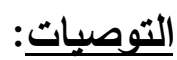
ا. مراعـاة المحكات التشخيصبة للدليل التشخيصسي الخـامس DSM-5 عند تتـخيص اضطراب الذاتوية r. إجراء مزيد من البحوث حول آليات التشخيص الدقيق لاضطراب الذاتوية

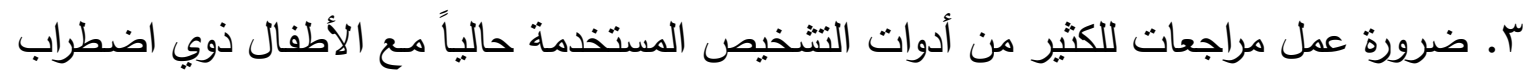

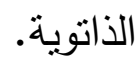

\section{البحوث المقترحة:}

1. النتخيص الفارق بين اضطراب الذاتوية واضطراب التواصل والتفاعل الاجتماعي.

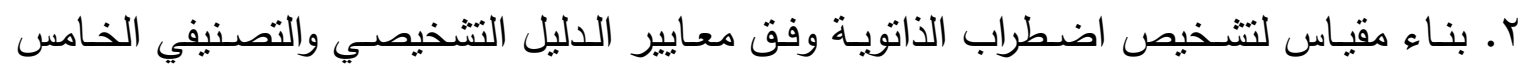
.DSM-5 r. أثر اختلاف أدوات التشخيص على نسبة انتشار اضطراب الذاتوية 


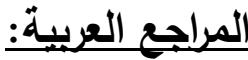

ا. الإمام (محمد)، الجوالده (فؤاد)، (• ( ץ): التوحد ونظريات العقل. عمان: دار النقافة للنشر

$$
\text { والتوزيع. }
$$

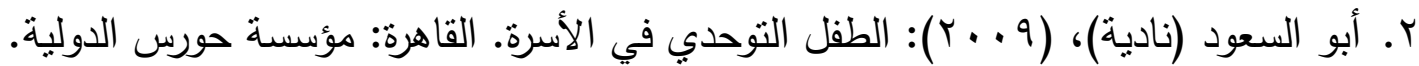

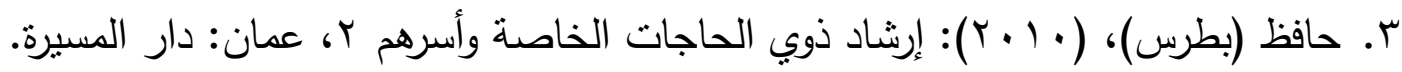

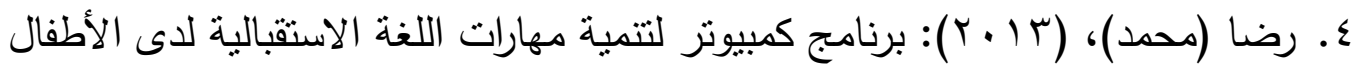
الذانويين، ماجستير ، كلبة التربية للطفولة المبكرة، جامعة القاهرة. ه. شريف، (السيد)، (ع ( †): مدخل إلى التربية الخاصة. القاهرة: دار الجوهرة للنشر والتوزيع.

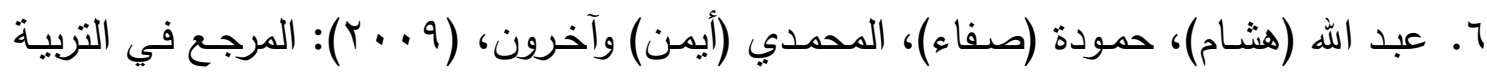
الخاصة. الرياض: مكتبة الثقري.

V. عوده (محمد)، (10 · ب): تشخيص وتتميـة مهارات الطفل الذاتوي. القـاهرة: مكتبـة الأنجلو المصرية.

^. هالاهان (دانيال)، كوفمان (جيمس)، (1 . ㄷ): سيكولوجية الأطفال غير العاديين وتعليمهم.

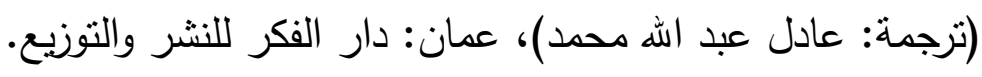

المراجي الأجنية:

9. American Psychiatric Association. (2000). Diagnostic and statistical manual of mental disorders (4th ed. Rev). Washington, DC: American Psychiatric Association.

10. American Psychiatric Association, (2013): Diagnostic and statistical manual of mental disorders (5th ed.). Arlington, VA: American Psychiatric Association.

11.Anderson George M, Montazeri Farhad,Bildt Annelies,(2015): Network Approach To Autistic Traits : Group and subgroup Analyses of ADOS Item Scores , J Autism Dev Disord, Vol 45 ,pp 3115-3132.

12.Barton, M. L., Robins, D. L., Jashar, D., et al, (2013): Sensitivity and specificity of proposed DSM-5 criteria for autism spectrum disorder in 
toddlers. Journal of Autism and Developmental Disorders, 43(5),pp: $1184-1195$

13.Beighley, J. S., \& Matson, J. L, (2014): Comparing social skills in children diagnosed with autism spectrum disorder according to the DSM-IV-TR and the DSM-5. Journal of Developmental and Physical Disabilities, 26, pp: 689-701.

14.Beighley, J. S., Matson, J. L., Rieske, R. D., and et al., (2013): Comparing challenging behavior in children diagnosed with autism spectrum disorders according to the DSM-IV-TR and the proposed DSM-5. Developmental Neurorehabilitation, 16(6), pp: 375-381.

15.Beighley, J. S., Matson, J. L., Rieske, R. D., et al, (2014): Differences in stereotypic behavior in adults diagnosed with autism spectrum disorders using the DSMIV- TR and the DSM-5. Journal of Developmental and Physical Disabilities, 26(2), 193-202.

16.Chakraborty Satabdi, Thomas Pramod, Bhatia Triptish et al , (2015): Assessment of Severity of Autism Using the Indian Scale for Assessment of Autism, Indian Journal of Psychological Medicine, Apr - Jun , Vol 37 , Issue 2, pp :169-174.

17.Emberti Gialloreti, Benvenuto Arianna, Battan Barbara, et al., (2016): Can biological components predict short-term evolution in Autism Spectrum Disorders? A proof-of-concept study, Journal of Pediatrics, 42:70, pp: 1-13.

18.Garg Shruti, Plasschaert Ellen, Descheemaeker Mie-Jef et al, (2015): Autism Spectrum Disorder Profile in Neurofibromatosis Type I, J Autism Dev Disord, 45, pp: 1649-1657.

19.Geier D. A.,Kerna J. K., Geier M.R.(2012) : Comparison of the Autism Treatment Evaluation Checklist (ATEC) and the Childhood Autism 
Rating Scale (CARS) for the Quantitative Evaluation of Autism, Journal of Mental Health Research in Intellectual Disabilities, 6 , pp :255-267, 20.George .B. Padmam. R, Nair. M. et al.,( 2014): CDC Kerala 11: Diagnosis of Autism Among Children Between 2 and 6 y - Comparison of CARS against DSM-IV-TR. Indian J Pediatr (December) 81(Suppl 2) pp:S125-S128.

21.Gibbs Vicki, Aldridge Fiona, Chandler Felicity. et al, (2012): Brief Report: An Exploratory Study Comparing Diagnostic Outcomes for Autism Spectrum Disorders Under DSM-IV-TR with the Proposed DSM-5 Revision. J Autism Dev Disord. 42, pp: 1750-1756.

22.Grant, R., \& Nozyce, M., (2013): Proposed changes to the American Psychiatric Association diagnostic criteria for autism spectrum disorder: Implication for young children and their families. Maternal and Child Health Journal, 17,pp : 586-592

23.Grzadzinski, R., Huerta, M., \& Lord, C. (2013): DSM-5 and autism spectrum disorders (ASDs): An opportunity for identifying ASD subtypes. Molecular Autism, 4, 12.

24.Hiller, R. M., Young, R. L., Weber, N., (2014): Sex differences in autism spectrum disorder based on dsm-5 criteria: Evidence from clinician and teacher reporting. Journal of Abnormal Child Psychology, 42,pp : 1381-1393

25.Kim, Y. S., Fombonne, E., Koh, Y. J., Kim, S. J., et al., (2014): A comparison of DSM-IV pervasive developmental disorder and DSM-5 autism spectrum disorder prevalence in an epidemiologic sample. Journal of the American Academy of Child and Adolescent Psychiatry, 53(5),pp : 500-508 
26.King, B. H., Veenstra-Vanderweele, J., \& Lord, C., (2013): DSM-5 and autism: Kicking the tires and making the grade. Journal of the American Academy of Child and Adolescent Psychiatry, 52, pp :454-457

27.King, B. H., Navot, N., Bernier, R., \& Webb, S. J., (2014): Update on diagnostic classification in autism. Current Opinion in Psychiatry, 27, pp: $105-109$.

28.Kite, D. M., Gullifer, J., \& Tyson, G. A., (2013): Views on the diagnostic labels of autism and Asperger disorder and the proposed changes in the DSM. Journal of Autism and Developmental Disorders, 43, pp: 1692-1700.

29.Kulage Kristine M., Smaldone Arlene M., Cohn Elizabeth G., (2014): How Will DSM-5 Affect Autism Diagnosis? A Systematic Literature Review and Meta-analysis, J Autism Dev Disord (2014) 44:1918-1932

30.MacFarlane, J. R., \& Kanaya, T., (2009): What does it mean to be autistic? Interstate variation in special education criteria for autism services. Journal of Child and Family Studies, 18(6),pp: 662-669

31.Maenner, M. J., Rice, C. E., Arneson, C. L., et al., (2014): Potential impact of DSM-5 criteria on autism spectrum disorder prevalence estimates. JAMA Psychiatry, 71(3),pp : 292-300

32.Malhi Prahbhjot \& Singhi Pratibha,(2015): Adaptive Behavior Functioning in Children with Autism, Indian J Pediatr (August) 82(8), pp : 677-681

33.Mattila, M. L., Kielinen, M., Linna, S. L, (2011): Autism spectrum disorders according to DSM-IV-TR and comparison with DSM-5 draft criteria: an epidemiological study. Journal of the American Academy of Child and Adolescent Psychiatry, 50(6), 583.pp: 511-592. 
34.Matson, J. L., Hattier, M. A., \& Williams, L. W. (2012): How does relaxing the algorithm for autism affect DSM-5 prevalence rates? Journal of Autism and Developmental Disorders, 42, pp: 1549-1556.

35.Mayes, S. D., Black, A., \& Tierney, C. D., (2013): DSM-5 underidentifies PDDNOS: Diagnostic agreement between the DSM-5, DSM-IV, and checklist for autism spectrum disorder. Research in Autism Spectrum Disorders, 7(2), pp :298-306

36.Mayes, S. D., Calhoun, S. L., Murray, M. Jet al., (2014): Final DSM-5 under-identifies mild autism spectrum disorder: Agreement between the DSM-5, CARS, CASD, and clinical diagnoses. Research in Autism Spectrum Disorders, 8(2), pp :68-73

37.Mazefsky, C., McPartland, J., Gastgeb, H., et al, (2013): Brief report: Comparability of DSM-IV and DSM-5 ASD research samples. Journal of Autism and Developmental Disorders, 43(5), pp: 1236-1242.

38.McPartland, J. C., Reichow, B., \& Volkmar, F. R., (2012): Sensitivity and specificity of proposed DSM-5 diagnostic criteria for autism spectrum disorder. Journal of de American Academy of Child and Adolescent Psychiatry, 51,pp: 368-383.

39.Neal, D., Matson, J. L., \& Hattier, M. A.et al, (2012): A comparison of diagnostic criteria on the autism spectrum disorder observation for children (ASD-OC). Developmental Neurorehabilitation, 15(5), pp: 329-335.

40.Prykanowski Debra A., Gage Nicholas A. Conroy Maureen A.,(2015) : Educational Implications of the DSM- 5 Criteria for Autism Spectrum Disorders.Beyond Behavior, Vol . 24, Iss. 2,pp:31-38

41.Rieske, R. D., Matson, J. L., Beighley, J. S., and et al., (2013): Comorbid psychopathology rates in children diagnosed with autism 
spectrum disorders according to the DSM-IV-TR and the proposed

DSM-5. Developmental Neurorehabilitation

42.Robles, R., Fresan, A., Evans, S. et al, (2014): Problematic, absent and stigmatizing diagnoses in current mental disorders classifications Results from the WHO-WPA and WHOIUPsyS Global Surveys. International Journal of Clinical and Health Psychology, 14,pp: $165-$ 177:

43.Rodriguez-Testal, J. F., Senin-Calderon, C. et al,(2014): From DSM-IVTR to DSM-5: Analysis of some changes.International Journal of Clinical and Health Psychology, 14,pp:221-231

44.Romero Marina, Aguilar Juan Manuel, Mejias Angel Del-Rey- et al, (2016): Psychiatric comorbidities in autism spectrum disorder: A comparative study between DSM-IV-TR and DSM-5 diagnosis. International Journal of Clinical and Health Psychology (2016) 16,pp: $266-275$

45.Rusu Cristina, Preda Cristina, Sireteanu Adriana et al. (2015) :Risk Factors in Autism Spectrum Disorders:The Role of Genetic,Epigentic.Immune and Environmental Interactions, Environmental Engineering and Management Journal April, Vol. 14, No. 4,pp: 901-917

46.Steensel Francisca J. A. , Bogels Susan M., de Bruin Esther I., (2015) : DSM-IV Versus DSM-5 Autism Spectrum Disorder and Social Anxiety Disorder in Childhood: Similarities and Differences. J Child FAM Stud, 24, pp: 2752-2756.

47.Taheri, A., Perry, A., \& Factor, D. C., (2014): Brief report: A further examination of the DSM-5 autism spectrum disorder criteria in practice. Journal on Developmental Disabilities, 21(1), pp: 116-121. 
48.Timini, S., (2014): No more psychiatric labels: Why formal psychiatric diagnostic systems should be abolished. International Journal of Clinical and Health Psychology, 14, pp: 208-215.

49.Turygin, N. C., Matson, J. L., Adams, H., et al, (2013): The effect of DSM-5 criteria on externalizing, internalizing, behavioral and adaptive symptoms in children diagnosed with autism. Developmental Neurorehabilitation, 16(4), 277-282.

50. Virues- Valkanova V, Rhodes F, Allan CL et al, (2013): Diagnosis and management of autism in adults, The Practitioner, Vol 257 , N 1761, pp 13-16.

51.Weitlauf, A. S., Gotham, K. O., Vehom, A. C., et al., (2014): Brief report: DSM-5 'levels of support' a component on discrepant conceptualizations of severity in ASD. Journal of Autism and Developmental Disorders, 44, pp: 471-476.

52.White, S. W., Smith, L. A., \& Schry, A. R., (2014): Assessment of global functioning in adolescents with autism spectrum disorders: utility of the Developmental Disability-Child Global Assessment Scale. Autism, 18, pp: 362-369.

53.Wiggins Lisa D., Reynolds Ann, Rice Catherine E. et al, (2015): Using Standardized Diagnostic Instruments to Classify Children with Autism in the Study to Explore Early Development. J Autism Dev Disord, 45, pp: $1271-1280$.

54.Wodrich, D. L., Pfeiffer, S. I., \& Landau, S., (2008): Contemplating the new DSM-5: Considerations from psychologists who work with school children. Professional Psychology: Research and Practice, 39,pp: 626632

55.Worley, J. A., \& Matson, J. L., (2012): Comparing symptoms of autism spectrum disorders using the current DSM-IV-TR diagnostic criteria 
and the proposed DSM-V diagnostic criteria. Research in Autism Spectrum Disorders, 6, 965-970.

56.Yong-Hwee Nah, Young Robyn L., Brewer Neil.(2014): Using the Autism Detection in Early Childhood (ADEC) and Childhood Autism Rating Scales (CARS) to Predict Long Term Outcomes in Children with Autism Spectrum Disorders. J Autism Dev Disord , 44:pp :2301-2310.

57.Young, R. L., \& Rodi, M. L., (2014): Redefining autism spectrum disorder using DSM-5: The implications of the proposed DSM-5 criteria for autism spectrum disorders. Journal of Autism and Developmental Disorders, 44(4), pp: 758-765. 